\title{
Modal sensing of efficient acoustic radiators with polyvinylidene fluoride distributed sensors in active structural acoustic control approaches
}

\author{
Robert L. Clark and Chris R. Fuller \\ Mechanical Engineering Department, Virginia Polytechnic Institute and State University, Blacksburg, \\ Virginia 24061
}

(Received 7 December 1990; revised 16 September 1991; accepted 12 February 1992)

\begin{abstract}
An experimental investigation was performed to determine the feasibility of implementing polyvinylidene fluoride (PVDF) piezoelectric distributed sensors on the surface of a structure as error sensors in an adaptive least-mean-squares $(\mathrm{lms})$ control approach to minimize acoustic radiation. While much research has been devoted to controlling vibration of structures with these sensors, they have yet to be implemented in structural acoustic control. To this end, two narrow strip PVDF sensors were positioned on a simply supported plate such that the dominant observed response was due to the odd-odd modes of the plate (i.e., the more efficient acoustic radiators). The error sensors in effect act as spatial wave-number filters and only observe those components that contribute significantly to far-field sound radiation. A variety of test cases were studied for controlling sound radiation due to a disturbance both on and off resonance. Results from these experiments indicate that PVDF sensors and piezoceramic actuators show much promise for controlling acoustic radiation from structures, to a large degree overcoming the need for error microphones in the far field.
\end{abstract}

PACS numbers: 43.40.Dx, 43.40.Vn

\section{INTRODUCTION}

The thrust of current research has been to develop intelligent structures that can adapt to their environment such that undesired vibration or acoustic radiation can be minimized. To this end, the adaptive least-mean-squares (lms) algorithm has played an important role in providing feed forward control approaches for narrow band applications. ${ }^{1-3}$ In addition, PZT (lead, zirconate, titanate) ceramic patches have been bonded to the surface of structures, or embedded within the structures to provide the necessary inputs for control of structural response as well as acoustic radiation. ${ }^{47}$ For structural control, PVDF (polyvinylidene fluoride) thin film has been implemented both as a sensor and a control actuator. ${ }^{8-11}$ Since the PVDF thin film can readily be shaped by etching or cutting strips of the material, a novel class of modal sensors and actuators have been developed. ${ }^{12}$ While much research has been devoted to applying modal sensing techniques for structural control, the technique is yet to be used for applications in structural acoustics.

The limitations experienced in control of acoustic radiation from structures are a result of the sensors applied in the control algorithm. For the most part, microphones have played a vital role as error sensors in both feedback and feed forward control approaches for structural acoustics. To develop a true adaptive structure, the sensing must become an integral part of the structure; therefore, the microphones located in the far field must somehow be eliminated. The purpose of this study is to extend the previous companion work conducted by the authors, which showed the validity of using multiple piezoelectric actuators for controlling sound radiation from structures, ${ }^{13}$ and replace the microphones used in the previous study with more favorable thin film PVDF sensors bonded to the surface of the structure. Preliminary results from this study suggest that PVDF thin film sensors can be shaped for specific structural acoustic control applications, acting as spatial "wave-number filters" in controlling radiation of structure-borne sound.

\section{THEORY}

\section{A. Sensor theory}

Since the far-field acoustic radiation is dependent on the structural response of the plate, driving the plate response to zero would certainly result in zero acoustic radiation. In practice, this would be impossible, since an infinite number of control actuators would be required to couple with the complex plate response. Fortunately, an alternative exists in structural acoustics. Structures such as beams, plates, and shells have modes of vibration which do not couple effectively to the acoustic media. As a result these modes do not efficiently radiate sound and thus do not require control.

Before developing the sensor theory, recall the response of a simply supported rectangular plate, which is given by

$w(x, y, t)=\sum_{m=1}^{\infty} \sum_{n=1}^{\infty} A_{m n} \sin \left(\frac{m \pi x}{L_{x}}\right) \sin \left(\frac{n \pi y}{L_{y}}\right) \exp (j \omega t)$,

where $\boldsymbol{m}$ and $n$ represent the modes in the $x$ and $y$ direction, respectively, $L_{x}$ and $L_{y}$ are the plate dimensions, $\omega$ is the frequency of excitation, and $A_{m n}$ are the modal amplitudes. The structural wave number is designated by

$$
k_{s}=\sqrt{\left(m \pi / L_{x}\right)^{2}+\left(n \pi / L_{y}\right)^{2}},
$$

and the well-known acoustic wave number is defined by

$$
k=\omega / c,
$$

where $\omega$ is the circular frequency and $c$ is the speed of sound in the media. For comparison, the radiation efficiency for several modes of the simply supported rectangular plate 


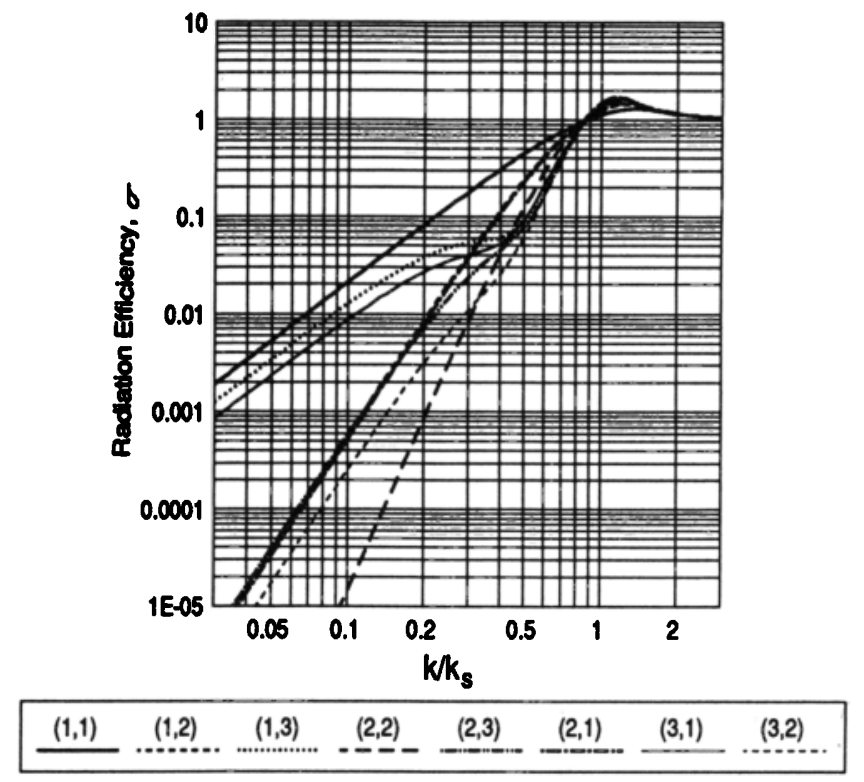

FIG. 1. Radiation efficiency of plate.

used in this study, with dimension of $380 \times 300 \times 1.96 \mathrm{~mm}$ thick, are presented graphically in Fig. 1 . Notice that the radiation efficiency approaches unity for all modes when the structural wave number is less than the acoustic wave number in the surrounding media. However, when the structural wave number is greater than the acoustic wave number, the radiation efficiencies for which both $m$ and $n$ are odd are the highest over most of the frequency range. ${ }^{14}$ The radiation efficiency of the even-even modes are the lowest, which is of interest in control of sound radiation in this regime, since these motions will not contribute significantly to the radiated field.

As a result of this observation, PVDF sensor shapes are chosen such that the even-even modes of the structure are not observed, reducing the dimensionality of the controller. Lee et al. previously developed an equation describing the closed-circuit charge signal $q(t)$ measured through the electrode of the PVDF lamina as follows: ${ }^{10}$

$$
\begin{aligned}
q(t)= & \left(\frac{h_{p}+h_{s}}{2}\right) \int_{0}^{L_{x}} \int_{0}^{L_{y}} \Gamma(x, y) \\
& \times\left(e_{31} \frac{\partial^{2} w}{\partial x^{2}}+e_{32} \frac{\partial^{2} w}{\partial y^{2}}+2 e_{36} \frac{\partial^{2} w}{\partial x \partial y}\right) d y d x,
\end{aligned}
$$

where $w$ is the plate response, $h_{p}$ is the piezoelectric lamina thickness, $h_{s}$ is the plate thickness, $e_{31}, e_{32}$, and $e_{36}$ are the piezoelectric field intensity constants, and $\Gamma(x, y)$ is the spatial function describing the shape of the sensor. In locating the sensors on the plate, one was placed vertically and the other horizontally as depicted in Fig. 2. The vertical sensor was located $52 \mathrm{~mm}$ from the left side of the plate and measured $22 \mathrm{~mm}$ wide, while the horizontal sensor was positioned $139 \mathrm{~mm}$ from the bottom side of the plate and also measured $22 \mathrm{~mm}$ wide. Since no skew angle is associated with polarization of the PVDF used in this study, $e_{36}=0$. The "spatial window" created by the sensors can be de-

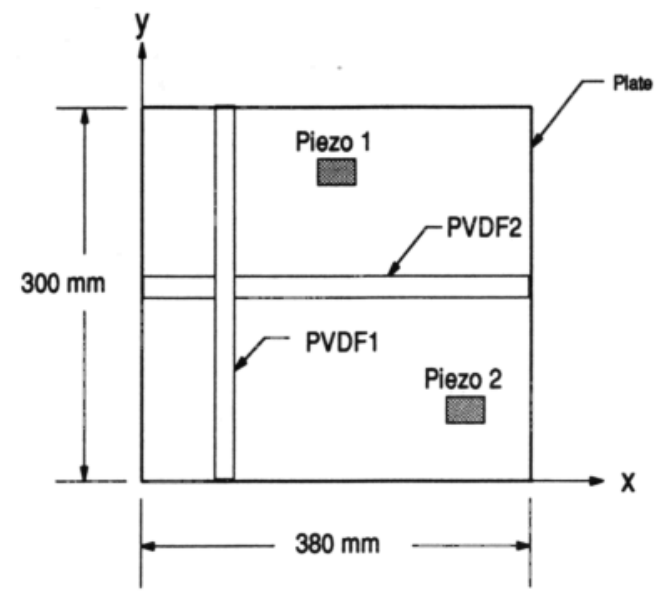

FIG. 2. Schematic of plate.

scribed in terms of step functions. For example, the function describing PVDF2 presented in Fig. 2 can be written as follows:

$$
\Gamma(x, y)=\left[u(x, y-c)-u\left(x-L_{x}, y-d\right)\right],
$$

where $u$ is a spatial step function in two dimensions, and the constants $c$ and $d$ are the coordinates locating the strip, 139 and $161 \mathrm{~mm}$, respectively. Substituting the equation for the response of the simply supported plate into the sensor equation along with the spatial function describing the sensor, we obtain

$$
\begin{aligned}
q(t)= & \left(\frac{h_{p}+h_{s}}{2}\right) \int_{0}^{L_{x}} \int_{0}^{L_{y}}[u(x, y-c)-u(x, y-d)] \\
& \times\left(\sum_{m=1}^{\infty} \sum_{n=1}^{\infty} \int\left[e_{31}\left(\frac{m \pi}{L_{x}}\right)^{2}+e_{32}\left(\frac{n \pi}{L_{y}}\right)^{2}\right]\right. \\
& \left.\left.\times A_{m n} \sin \left(\frac{m \pi x}{L_{x}}\right) \sin \left(\frac{n \pi y}{L_{x}}\right) \exp (j \omega t)\right\}\right) d x d y,
\end{aligned}
$$

which can be integrated over the surface. Upon evaluating the integral, the response can be simply expressed as

$$
\begin{aligned}
q(t)= & \frac{h_{p}+h_{s}}{2} \sum_{m=1}^{\infty} \sum_{n=1}^{\infty} A_{m n}\left[e_{31}\left(\frac{m L_{y}}{n L_{x}}\right)+e_{32}\left(\frac{n L_{x}}{m L_{y}}\right)\right] \\
& \times[\cos (m \pi)-1]\left[\cos \left(\frac{n \pi d}{L_{y}}\right)-\cos \left(\frac{n \pi c}{L_{y}}\right)\right] .
\end{aligned}
$$

As is obvious from this expression, for even $m$, the integral is zero; therefore, all even modes in the $x$ direction are not observed by this sensor. A similar formulation for the vertical sensor results in rejection of even modes in the $y$ direction for that sensor. Since two sensors are implemented to measure the odd modes in the $x$ and $y$ direction, contributions of odd-even modes result in the error signal as well as odd-odd modes. The least efficient even-even modes are therefore not observed. Each signal is weighted equally in the lms algorithm, and as a result, the odd-even modes are weighted to a lesser degree than the odd-odd modes since only one sensor can detect the mode as opposed to two. Further work will 
consider more specialized shape functions for the sensor which will discriminate between different modes.

\section{B. Wave-number analysis}

Before discussing the experiment and the corresponding results, it is appropriate to introduce the method used to interpret the results. The typical approach used in describing the acoustic field based on the structural response is to compare the modal amplitudes before and after control. At this point two terms will be defined to describe the methods of control, "modal suppression" and "modal restructuring." Modal suppression is the most obvious method of control, and it is typically associated with on-resonance cases. In this case, after minimizing the acoustic field, the modal amplitudes and thus the panel response fall, resulting in a reduction in the acoustic radiation.

The second method of control, modal restructuring, is the most interesting and complicated of the two methods defined. Modal restructuring is associated with an increase in modal amplitudes and a change in the relative phasing among the "uncontrolled" modes such that the panel has a lower overall radiation efficiency. An alternate interpretation is based upon mathematically viewing the system response (vibration and acoustic) as a superposition of " modal" components. In this case, it is convenient to talk in terms of the uncontrolled modes although the closed loop system has new modes and resonant frequencies. ${ }^{15}$ To clarify this method, a simple diagram will be used to aid in the discussion. For simplicity the panel response will be assumed to be composed of two modes, the $(1,1)$ and the $(2,1)$ mode of the structure, depicted in the $x$-z plane of Fig. 3. Both the uncontrolled and controlled acoustic responses are presented below the schematic of the structural modes. The resulting sound field is a superposition of the sound pressures contributed by each structural mode. In the uncontrolled case, the acoustic response of the $(2,1)$ mode is observed to reinforce that of the $(1,1)$ mode at the location of the error microphone. After applying control, the modal amplitude of the

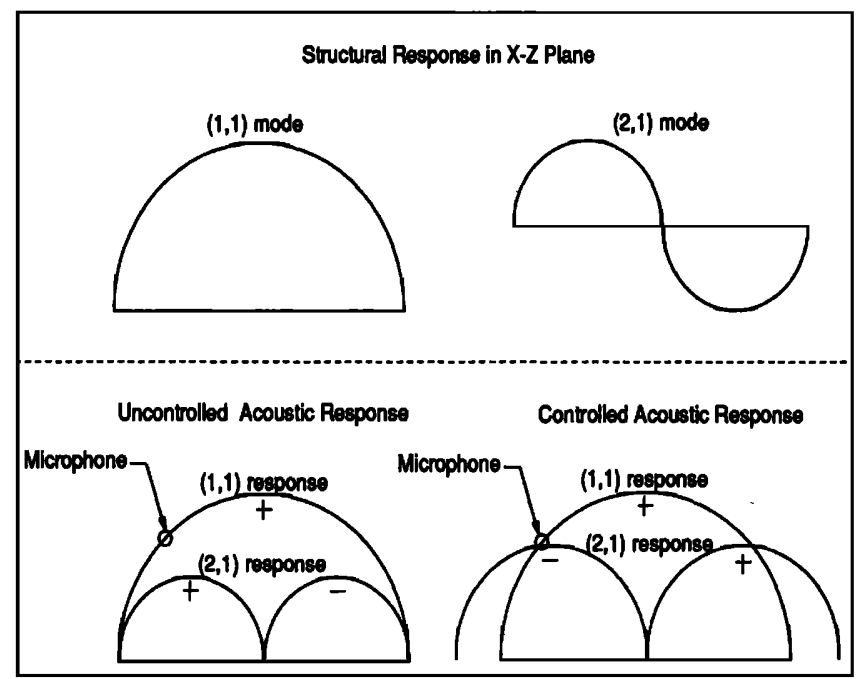

FIG. 3. Example of acoustic and structural modes.
$(2,1)$ mode is increased such that the acoustic response resulting from the $(2,1)$ mode is equivalent to that of the $(1,1)$ mode; however, the phase between the modes is shifted such that the acoustic response decreases at the error microphone. Alternatively, the increase in structural response of the $(2,1)$ mode has led to a residual structural response that has a lower overall radiation efficiency. For the simple contrived case described here, the results are readily interpreted, but as the number of modes included is increased, physical interpretation of the modal amplitudes is impossible other than to observe that some form of modal restructuring has occurred.

To alleviate this problem of interpretation, an alternative method of analyzing the data will be introduced. This method is based on a wave-number transform, which is analogous to obtaining the frequency spectrum from a time-dependent signal. ${ }^{14}$ To obtain the wave-number transform, a Fourier integral transform of the spatial response is evaluated:

$$
\begin{aligned}
F\left(k_{x}, k_{y}\right)= & \int_{-\infty}^{+\infty} \int_{-\infty}^{+\infty} f(x, y) \exp \left(-j k_{x} x\right) \\
& \times \exp \left(-j k_{y} y\right) d y d x .
\end{aligned}
$$

Replacing $f(x)$ with the spatial response of the plate (1) and evaluating the integral over the boundaries of the plate, the wave-number transform is obtained for each mode as

$$
\begin{aligned}
W_{m n}\left(k_{x}, k_{y}\right)= & A_{m n} \int_{0}^{L_{x}} \int_{0}^{L_{y}} \sin \left(\frac{m \pi x}{L_{x}}\right) \sin \left(\frac{n \pi y}{L_{y}}\right) \\
& \times \exp \left(-j k_{x} x\right) \exp \left(-j k_{y} y\right) d y d x .
\end{aligned}
$$

Plotting the magnitude of this function yields physical insight into the structural acoustic response. An idealized example of a wave-number spectrum is presented in Fig. 4 to convey the concepts. The acoustic wave number, presented in Eq. (3), is the critical number defining the region between structural wave-number components that do not radiate acoustic energy and components that simply create nearfield acoustic disturbances. The radiating region is termed the supersonic region and is the shaded region in Fig. 4 where the structural wave number is less than the acoustic
Uncontrolled

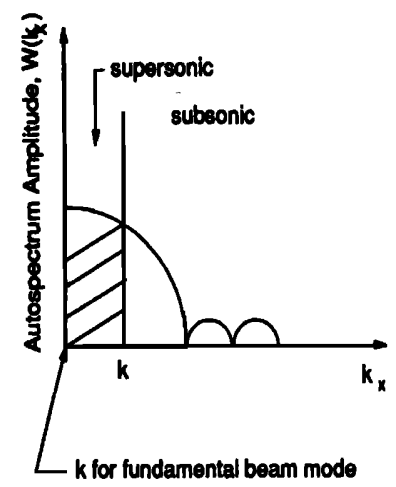

Controlled

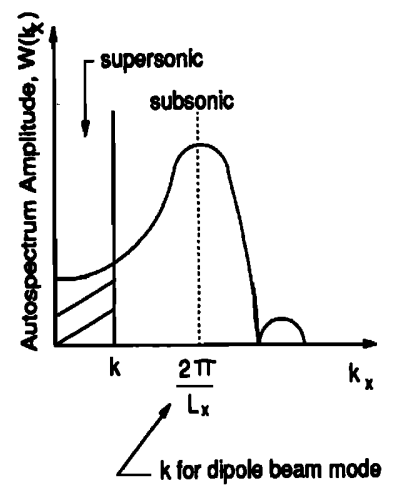

FIG. 4. Example of $k$-plane results. 
wave number. ${ }^{14}$ The nonradiating region is termed the subsonic region and is defined when the structural wave number is greater than the acoustic wave number.

Using this technique to describe the relationship between the structural and acoustic response of the plate facilitates interpretation of results since the uncontrolled and controlled plate motions are being decomposed into wave components. For the case of modal suppression, the overall magnitude of the wave-number transform is simply reduced after applying control. However, for the case of modal restructuring, the energy is observed to shift from the supersonic (radiating) region to the subsonic (nonradiating) region as illustrated in Fig. 4. (The example of Fig. 4 is for a 1-D system.) For the case presented, a cross section of the wave-number transform was illustrated for simplicity. In reality, a three-dimensional figure, including negative wavenumber components, is required to illustrate the results since the structural wave number is a function of both the $x$ and $y$ direction as illustrated in Eq. (2).

\section{EXPERIMENTAL SETUP AND PROCEDURE}

The experimental setup was identical to that described previously by the authors in the companion paper ${ }^{13}$ with the exception of the sensors used in the cost function of the control algorithm. The PVDF thin film sensors described in the previous section were attached to the structure with doublesided tape and electrical leads were attached with copper tape to the top and bottom surface of the PVDF elements. A picture of the test plate is presented in Fig. 5 with both PZT actuators and PVDF sensors attached to the surface.

The electrical response of each sensor was conditioned with matched high-impedance (i.e., Giga ohms) operational amplifiers, and gains were provided with noninverting amplifiers. The input impedance of the amplifiers must be much greater than that of the sensors to prevent loading of the PVDF sensors. The thin film sensors used in this study measured $28 \mu \mathrm{m}$ thick, and material properties can be found in the Kynar Piezo Film Technical Manual. ${ }^{16}$ It is worth mentioning that the sensors have a favorable direction of polarization and hence $e_{31}$ is approximately twice as great as $e_{32}$. Based on this observation, the sensors were oriented either

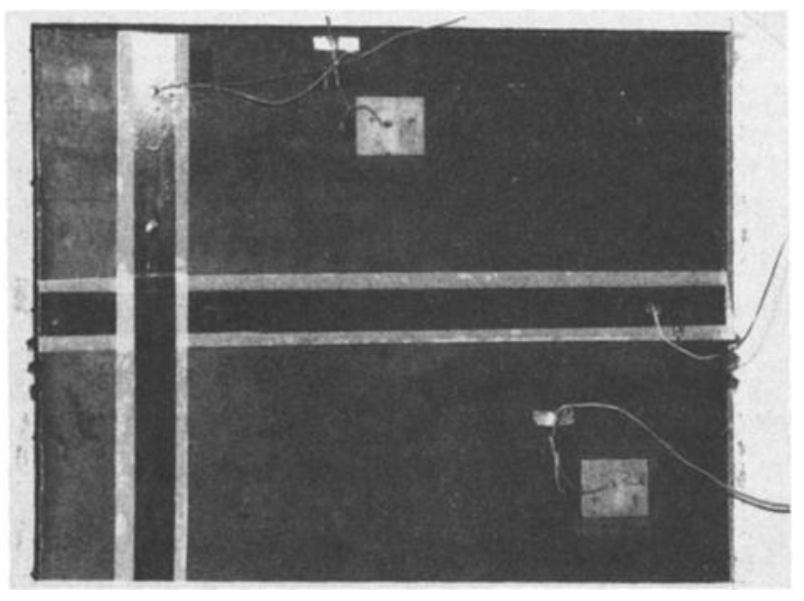

FIG. 5. Plate configured with PZT actuators and PVDF sensors.
TABLE I. Theoretical versus experimental resonant frequencies.

\begin{tabular}{ccc}
\hline \hline Mode & Theoretical $f_{m n}(\mathrm{~Hz})$ & Experimental $f_{m n}(\mathrm{~Hz})$ \\
\hline$(1,1)$ & 88 & 88 \\
$(2,1)$ & 189 & 187 \\
$(1,2)$ & 250 & 253 \\
$(2,2)$ & 351 & 349 \\
$(3,1)$ & 357 & 353 \\
$(1,3)$ & 519 & 511 \\
$(3,2)$ & 520 & 522 \\
$(4,1)$ & 592 & 584 \\
$(2,3)$ & 621 & 612 \\
\hline \hline
\end{tabular}

horizontally or vertically, depending on the application, to take advantage of the more favorable direction of sensitivity. The plate was also grounded to the common ground of the instrumentation to eliminate electrical "cross talk" between the PZT actuators and the PVDF sensors.

In conducting the experiments, the disturbance input was achieved with a shaker attached to the plate with a stinger at spatial coordinates of $(240,130) \mathrm{mm}$ for the onresonance cases and $(170,100) \mathrm{mm}$ for the off-resonance cases. The shaker was driven harmonically, and before applying control, both the acoustic response and structural response were quantified as outlined previously by the authors ${ }^{13}$ to provide a basis for comparison. Control was achieved with the multichannel version of the filtered- $X$ adaptive $1 \mathrm{~ms}$ algorithm as discussed in the companion paper ${ }^{13}$ and control inputs were supplied with the piezoelectric actuators depicted in Fig. 2. Error information was obtained from the electrical response of the PVDF thin film sensors to create the necessary control cost function. Upon achieving control, the acoustic and structural response were again quantified to evaluate the level of acoustic attenuation as well as the physical mechanism of control. For future reference, the predicted and measured resonant frequencies of the plate are given in Table I. As indicated, the measured values are within $1 \%$ for the modes presented. Greater details of the plate mode shapes as well as specifics required to measure both the acoustic response and structural response of the plate are given in the related study by the authors. ${ }^{13}$

\section{RESULTS}

Initial experiments were conducted implementing the PVDF thin film error sensors in the control algorithm. Both on and off resonance cases are presented to compare sound attenuation for up to two channels of control. After completing these tests, microphones were implemented as error sensors in the control algorithm for the same input disturbance location and control actuator configuration to provide a basis for comparison. Finally, example results from the wavenumber analysis are presented which illustrate the mechanisms of control.

\section{A. Comparison between microphone and PVDF thin film error sensors}

For the first case, the plate was driven on-resonance for the $(1,1)$ mode. As illustrated by the acoustic response pre- 


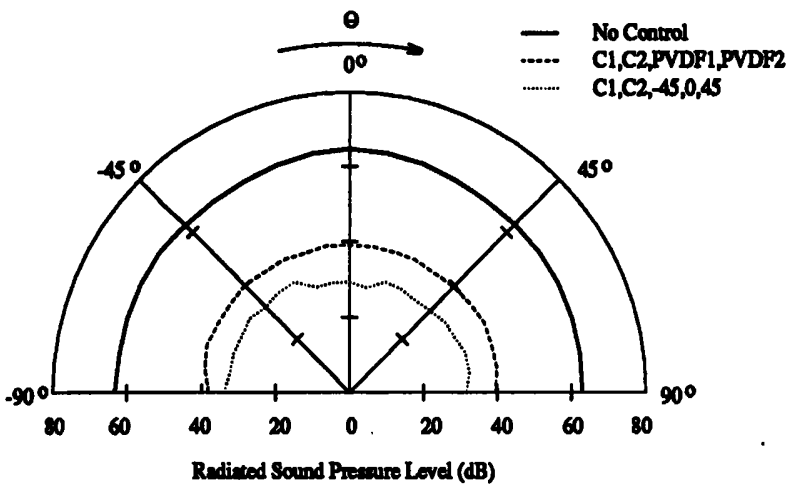

(a) Acoustic Directivity Pattern

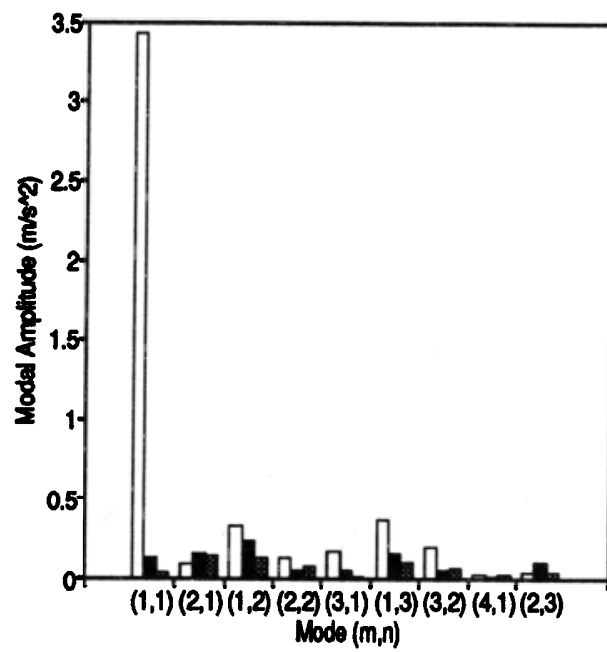

(b) Modal Amplitudes

FIG. 6. Response at $(1,1)$ mode (PVDF error sensors).

sented in Fig. 6(a), sound attenuation on the order of $30 \mathrm{~dB}$ was obtained. In the legend of the figure, " $\mathrm{C} \#$ "' indicates the piezoelectric actuator used in the control implementation and "PVDF\#" indicates the chosen PVDF error sensor. Each of the numbered elements is depicted in the plate schematic of Fig. 2. Considering the modally decomposed structural response, presented in Fig. $6(\mathrm{~b})$, the $(1,1)$ mode of the plate is obviously dominant before control. After applying control, a modal suppression occurred, whereby the amplitudes of all modes have decreased. ${ }^{17}$ (Later results will demonstrate how this concept can be understood from the wavenumber point of view.) As expected, the response of the $(1,1)$ mode was dominant before control, and sound attenuation was achieved by reducing the structural modal response by approximately $30 \mathrm{~dB}$.

For comparison, microphones located at $+45^{\circ}, 0^{\circ}$, and $-45^{\circ}$ were implemented as error sensors as indicated in Fig. 6(a), and the same control experiment was conducted on-

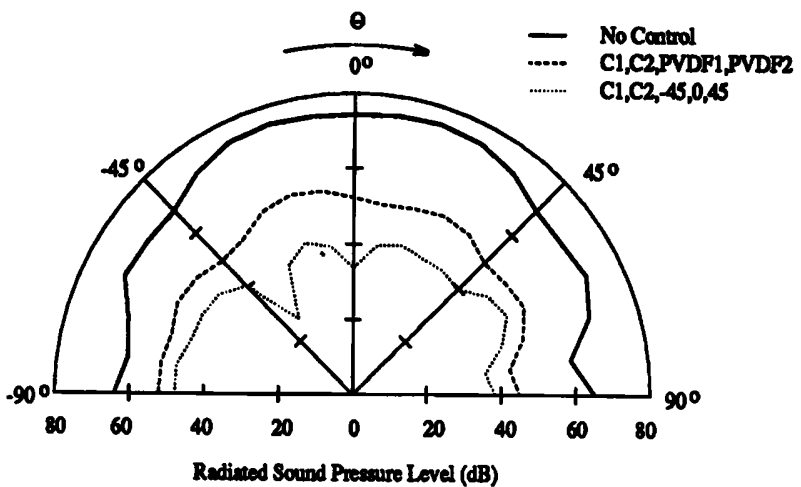

(a) Acoustic Directivity Pattern
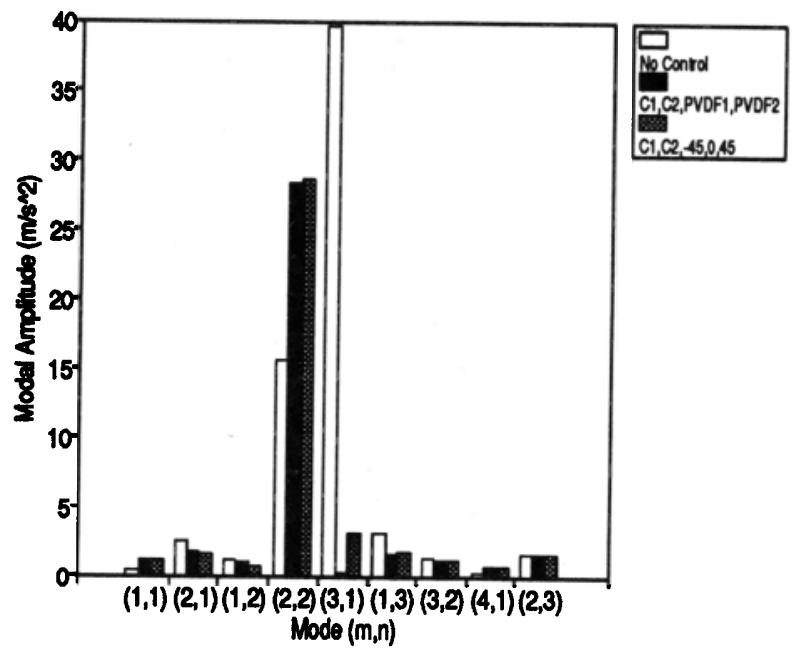

(b) Modal Amplitudes

FIG. 7. Response at $(3,1)$ mode (PVDF error sensors).

resonance for the $(1,1)$ mode of the plate. In the legend of the figure, the microphone position is given in units of degrees with the control actuator number used in the experiment. The controlled response when implementing the microphone error sensors resulted in sound attenuation on the order of 35 to $40 \mathrm{~dB}$, an increase in attenuation of 5 to $10 \mathrm{~dB}$ over that observed when utilizing the PVDF error sensors. This increased level of attenuation can be attributed to the further reduction of the $(1,1)$ mode observed when minimizing the response of the microphone error sensors as is apparent in Fig. 6(b).

As a second case, the plate was driven slightly aboveresonance (i.e., $355 \mathrm{~Hz}$ ) for the $(3,1)$ mode. Upon minimizing the response of the two PVDF error sensors, the acoustic response was attenuated by approximately $20 \mathrm{~dB}$ as can be seen in Fig. 7(a). Results from the modal decomposition indicate that control was achieved by modal suppression of the dominant $(3,1)$ mode with an increase in the response of 
the $(1,1),(2,2)$, and $(4,1)$ structural modes as indicated in Fig. 7(b). The control spillover into the $(2,2)$ mode has little effect on the structural acoustic response due to the low radiation efficiency of this mode at $k / k_{s}=0.25$; however, the small increase in response of the $(1,1)$ mode limits the level of sound attenuation since the radiation efficiency of this mode is approximately 10 times greater than that of the $(3,1)$ mode.

The microphone error sensors located at $+45^{\circ}, 0^{\circ}$, and $-45^{\circ}$ were again implemented as error sensors as indicated in Fig. 7(a), and the same control experiment was conducted near the resonant frequency of the $(3,1)$ mode with the two identical control actuators used in the previous test. The resulting acoustic response after control was observed to decrease by approximately $30 \mathrm{~dB}$ as indicated in Fig. 7(a). While the general shape of the directivity patterns resulting from control with either microphone error sensors or PVDF error sensors is similar, greater levels of acoustic attenuation resulted when implementing the microphone error sensors as evident in Fig. 7(a). Referring to the modal response presented in Fig. 7(b), control was achieved in this case by two mechanisms. First of all, the dominant $(3,1)$ mode, which is an efficient acoustic radiator, was reduced (modal suppression). However, some increase in the amplitude of the $(2,2)$ mode was observed. As discussed earlier, this spillover into the $(2,2)$ mode has little effect on the level of sound radiation since the radiation efficiency of this mode is approximately 15 times less than that of the $(3,1)$ mode for $k / k_{s}=0.25$ as can be seen in Fig. 1. Upon comparing the structural modal response when applying control with the PVDF error sensors to that when applying control with the microphone error sensors, one observes that the controlled modal amplitudes are nearly identical with the exception of the $(3,1)$ mode. Less attenuation of the $(3,1)$ mode occurred when using the microphone error sensors. Upon achieving control, the $(3,1)$ mode was reduced; however, upon reaching a level where the sound radiation from the $(1,1)$ mode and $(3,1)$ mode were comparable, the phase relationship between the modes was restructured such that the $(3,1)$ mode and $(1,1)$ mode were out of phase with each other, explaining the increased level of attenuation observed when implementing the microphone error sensors.

The final test was conducted off-resonance at a driving frequency of $320 \mathrm{~Hz}$, which lies between resonant frequencies corresponding to the $(1,2)$ and $(2,2)$ modes of the structure. Results from the acoustic response before and after control are presented in Fig. 8(a). As observed, approximately $15 \mathrm{~dB}$ of global sound attenuation was obtained after minimizing the response of the PVDF distributed sensors. The modal structural response of the plate was observed to decrease at every mode under control conditions, with the exception of the $(1,2),(1,3)$, and $(4,1)$ modes as indicated in Fig. 8(b). Of these three modes, the $(1,2)$ mode is of primary concern since the modal amplitude after control is of a comparable level with that of the $(3,1)$ mode before control. Fortunately, the radiation efficiency of the $(3,1)$ mode is approximately 3 times greater than that of the $(1,2)$ mode for $k / k_{s}=0.25$, hence the spillover into the $(1,2)$ mode does not prevent attenuation of the radiated sound.

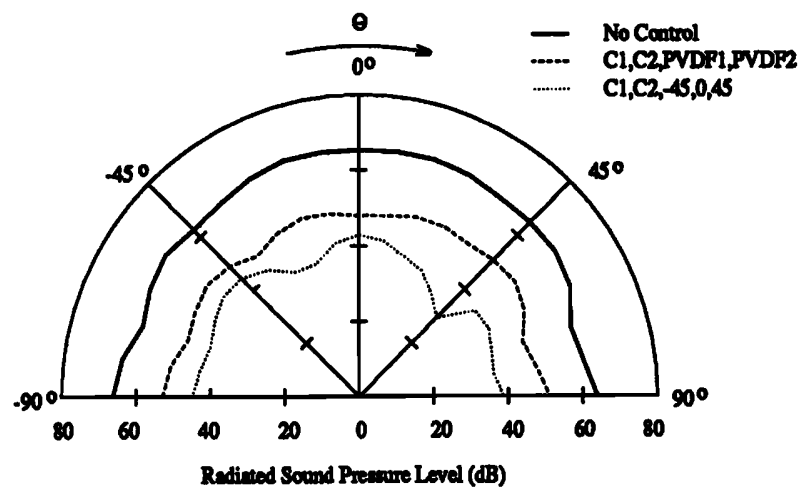

(a) Acoustic Directivity Pattern

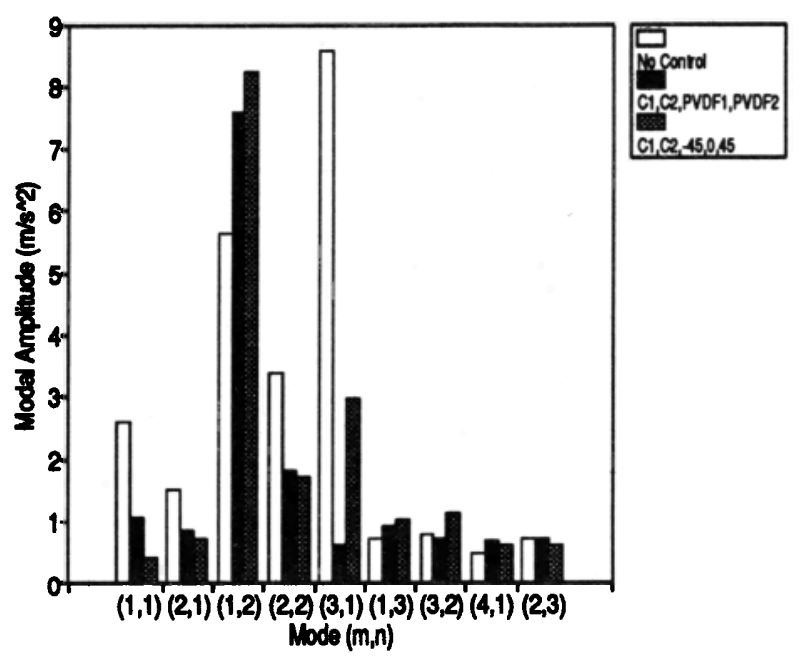

(b) Modal Amplitudes

FIG. 8. Response at $320 \mathrm{~Hz}$ (PVDF error sensors).

For comparison, the structure was driven off-resonance at an excitation frequency of $320 \mathrm{~Hz}$. Microphones positioned at $+45^{\circ}, 0^{\circ}$, and $-45^{\circ}$ were again utilized as error sensors, and two channels of control were implemented. As can be seen in Fig. 8(a), levels of sound attenuation on the order of $20 \mathrm{~dB}$ resulted. It is apparent from Fig. 8(b) that control was achieved through modal suppression of the $(3,1)$ and $(1,1)$ mode as well as modal restructuring between these modes. As demonstrated in the on-resonance test case presented in Fig. 7 , the $(3,1)$ mode and $(1,1)$ mode were phased for destructive interference upon achieving control. This again serves to explain why the amplitude of the $(3,1)$ mode was attenuated less when implementing the microphone error sensors than when implementing the PVDF error sensors. Since the radiation efficiency of the $(1,1)$ mode is approximately 10 times greater than that of the $(3,1)$ mode, the modal response of the $(3,1)$ mode must be greater than that of the $(1,1)$ mode for destructive inter- 
ference to occur (with the appropriate phase relationship). The residual plate response thus had a lower overall radiation efficiency upon achieving control with the microphone error sensors.

\section{B. Wave-number analysis}

The wave-number approach is introduced to facilitate interpretation of the relationship between the structural response and the corresponding acoustic response of the plate. Recalling the results corresponding to excitation of the $(1,1)$ mode, presented in Fig. 6(b), the modal amplitude of the dominant $(1,1)$ mode was significantly reduced regardless of whether PVDF or microphone error sensors were used. As an alternative method of analysis, performing a wavenumber transform of the structural response corresponding to the uncontrolled and controlled response for this test case will be examined. Before control, the largest amplitudes (or velocities since the response is harmonic) are observed in the supersonic region of the wave-number transform presented in Fig. 9. Only a "slice" of the $k$ transform (since $k_{y}$ was set equal to zero) was presented in the graphs to help clarify the method of analysis. In analyzing the results, a two-dimensional $k$ transform was performed, and the trends were the same as those presented in the following one-dimensional $k$ transforms. The one-dimensional graph was chosen simply to make visualization of the process easier for the reader. For an excitation frequency of $87 \mathrm{~Hz}$, the acoustic wave number is 1.6. After applying control with PVDF error sensors, the amplitudes in the supersonic region (radiating region) decreases as was the case in the subsonic region (nonradiating region). As a result, the acoustic radiation of the structure decreased since the total energy radiated was reduced upon applying control. As described earlier, this method of control was termed modal suppression. The wave-number transform corresponding to the controlled response implementing microphone error sensors is very similar; however, greater levels of attenuation are observed in the supersonic region of the wave-number transform, explaining the increased level of acoustic attenuation.

In the second test case, the plate was driven slightly

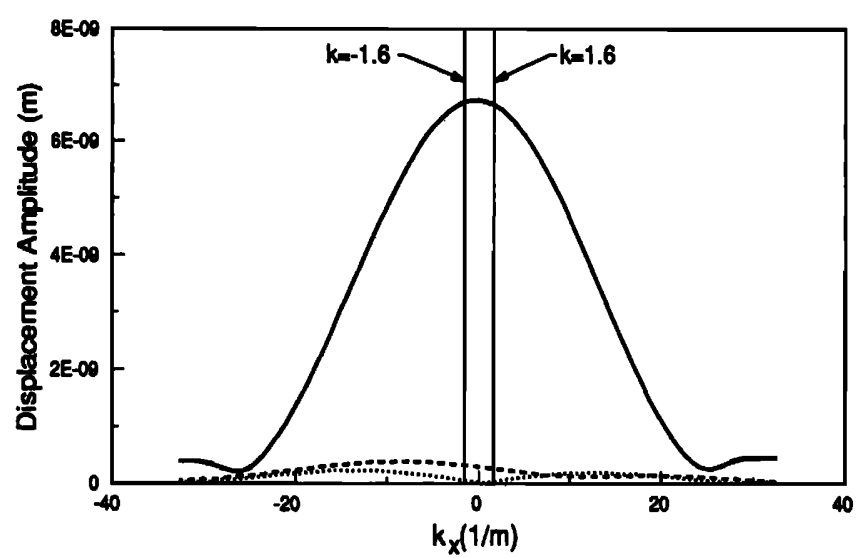

No Control PVDF Sensors Mlcrophone Sensors

FIG. 9. The $k$ transform $\left(k_{y}=0\right)$ for plate driven at $(1,1)$ mode.

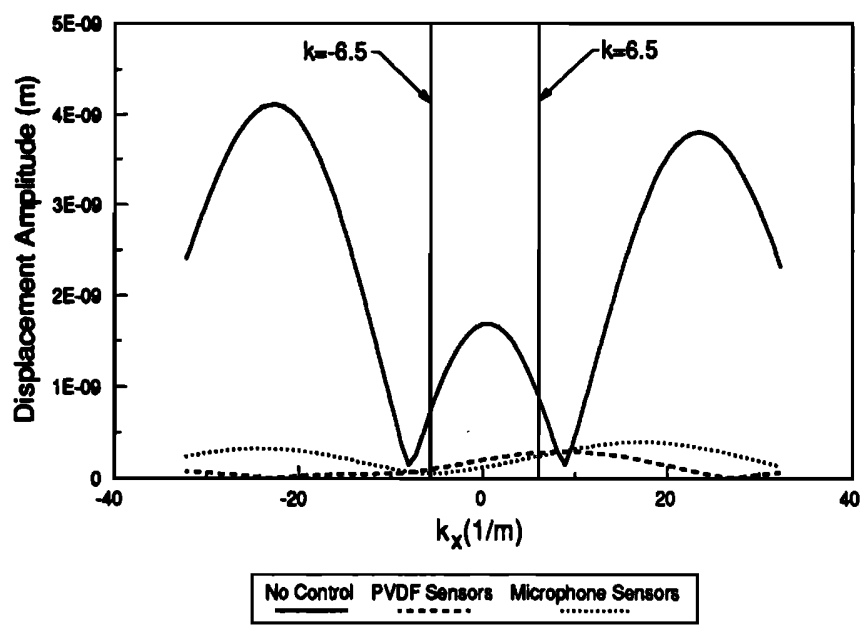

FIG. 10. The $k$ transform $\left(k_{y}=0\right)$ for plate driven at $(3,1)$ mode.

above resonance for the $(3,1)$ mode with an acoustic wave number of 6.5. The wave-number transform corresponding to the uncontrolled and controlled response is presented in Fig. 10. As evident, the response in the supersonic region of the wave-number transform was significantly reduced when using either PVDF or microphone error sensors; however, a greater level of attenuation was observed when implementing the microphone error sensors. In the subsonic region, the amplitudes of the spectrum are greater when applying control with the microphone error sensors than that observed when applying control with the PVDF error sensors. Recalling the results presented in Fig. 7(a), greater levels of sound attenuation were observed when implementing the microphone error sensors. This illustrates that the modal restructuring required to achieve greater levels of sound attenuation with microphone error sensors serves to increase spillover into the subsonic region of the wave-number spectrum in comparison to that resulting from control with PVDF error sensors. This spillover can be attributed to the differences noted in the modal response of the $(3,1)$ mode corresponding to the two control cases.

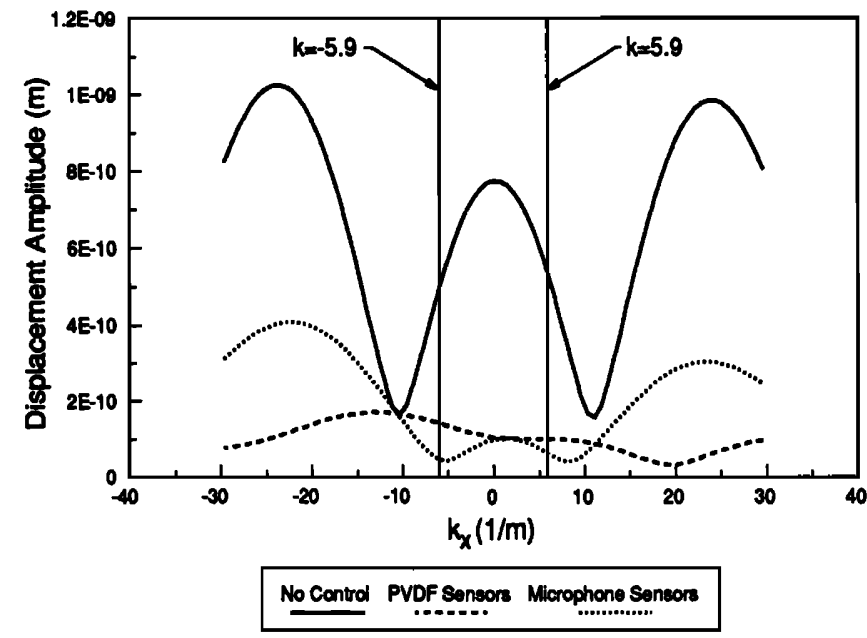

FIG. 11. The $k$ transform $\left(k_{y}=0\right)$ for plate driven at $320 \mathrm{~Hz}$. 
In the final example, consider the results presented in Fig. 8, corresponding to excitation of the plate off resonance at $320 \mathrm{~Hz}$. The acoustic wave number in this case is approximately 5.9. The amplitudes of the $k$ transform were significantly decreased as illustrated in Fig. 11, and the method of control is again predominantly described by modal suppression whether PVDF or microphone error sensors were implemented in the control approach. This result was also observed in Fig. 8(b) with the exception of an increase in the amplitude of the $(1,2)$ mode of the structure, resulting from control spillover. As indicated in Fig. 11, whether PVDF or microphone error sensors were implemented in the control approach, the amplitude of the wave-number spectrum in the supersonic region as well as the subsonic region decreased. However, when implementing the microphone error sensors, the amplitude of the wave-number spectrum was significantly less in the supersonic region than in the subsonic region compared to the controlled response when utilizing the PVDF error sensors. The greater levels of attenuation in sound radiation can thus be attributed to the spillover into the subsonic region of the wave-number spectrum. The structural wave number of the $(3,1)$ mode is 27 , which is approximately the location of the two maxima corresponding to the controlled response when implementing the microphone error sensors. As indicated in Fig. 8(b), the controlled response when implementing microphone error sensors resulted in less attenuation of the $(3,1)$ mode than that resulting from applying control with the PVDF error sensors. The phasing between the $(3,1)$ mode and $(1,1)$ mode serves to further reduce the response in the supersonic region at the expense of less attenuation in the subsonic region.

\section{Summary}

While significant levels of sound attenuation were obtained when implementing the PVDF error sensors for both on- and off-resonance test cases, greater levels of sound attenuation were observed when implementing the microphone error sensors. This results from the fundamental differences in the two sensors. The PVDF error sensors weight each structural mode as a function of their shape and position on the structure (i.e., spatial window created by the sensors) and as a function of the modal indices since the response is proportional to the integral of strain over the surface of application as evident in Eq. (7). Hence, the sensor becomes increasingly responsive to higher-order modes; however, the response of these modes are filtered due to the frequency response characteristics of the structure. When the controlled modal amplitudes of the dominant lower-order modes (for structural acoustic control) are of comparable levels with the higher-order modes, the PVDF sensor "shifts interest" to these higher-order modes. This explains why the amplitude of the $(3,1)$ mode was always reduced to a level comparable to that of the $(1,1)$ mode. The $(3,1)$ mode is weighted approximately three times greater than that of the $(1,1)$ mode since the sensor yields an electrical response proportional to the integral of strain over the surface of application.

In contrast, the microphone error sensors obviously in- clude the structural acoustic transfer function and hence are ideal error sensors for this application. The radiation efficiency of each mode is automatically included in the cost function since the electrical response of the microphone is sensitive to the resulting sound radiated from each structural mode and not the amplitudes of the structural modes. This serves to explain why the $(3,1)$ mode was attenuated less when implementing microphone error sensors than when implementing the PVDF error sensors. As the response of the $(3,1)$ mode was reduced to a level where the radiation efficiency was comparable to that of the $(1,1)$ mode, the phase relationship between the two modes was simply configured for descriptive interference (i.e., out of phase) as discussed earlier by the mechanisms conveyed in Fig. 3. When implementing the PVDF error sensors, the $(3,1)$ mode was observed to be out of phase with the $(1,1)$ mode; however, the amplitude was reduced to a level where destructive interference of the acoustic response was not possible since the PVDF sensor is sensitive to the integral of strain over the surface of application.

\section{CONCLUSIONS}

Experiments have been performed that consider the use of distributed PVDF error sensors in an active structural acoustic control approach. The PVDF sensors were attached directly to the plate and shaped/located to observe only particular structural motion. In general, the results show good attenuation of radiated sound levels for both onor off-resonance response with a two channel control approach. Sound attenuation on the order of 20 to $30 \mathrm{~dB}$ was achieved for the on-resonance cases and approximately 15 $\mathrm{dB}$ of sound attenuation was obtained for the off-resonance condition; however, greater levels of attenuation were expected for on-resonance conditions since the majority of the acoustic response is due to a single mode of the structure. The PVDF error sensors weight each structural mode as a function of their shape and position on the structure (i.e., spatial window created by the sensors) and as a function of the modal indices since the response is proportional to the integral of strain over the surface of application. This serves to limit the level of control spillover and suppress the response of the higher-order modes of the structure. However, the level of modal restructuring which can be achieved is reduced, limiting the level of acoustic attenuation. The obvious design objective would be a compromise between the above observations whereby the PVDF strip sensors are optimally positioned on the structure to effectively weight the structural modes. This effect is so complex that it may only be accomplished for high modal densities in conjunction with optimal design techniques, ${ }^{18}$ which is the basis for future work.

Several techniques were used to study the results and obtain the previous conclusions. Due to the complexity of the structural response before and after control, the wavenumber transform was chosen as the most efficient method of analysis in discovering the relationship between the structural response and the acoustic response. Two methods of control were observed, modal suppression and modal restructuring. In modal suppression, the amplitudes of the 
structural response were observed to decrease for all modes present. However in modal restructuring, control is achieved by spillover of control energy into the plate modes such that the residual response has a lower overall radiation efficiency and an associated lower radiation of acoustic power. In both cases, the amplitudes in the supersonic region of the wave-number transform of the structural response decreased, corresponding to a reduction in the total far-field sound radiation from the structure. In future work, this method of analysis and terminology will be used to study and describe the physical nature of structural-acoustic control.

\section{ACKNOWLEDGMENT}

This work was supported by DARPA/ONR under Grant No. ONR-N00014-88-K-0721.

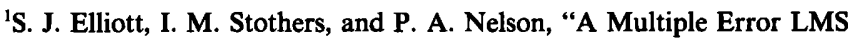
Algorithm and its Application to the Active Control of Sound and Vibration," IEEE Trans. Acoust. Speech Signal Process. ASSP-35 (1), 14231434 (1987).

${ }^{2}$ C. R. Fuller, G. P. Gibbs, and R. J. Silcox, "Simultaneous Active Control of Flexural and Extensional Waves in Beams," J. Intelligent Mater. Systems Struct. 1 (2), 235-247 (1990).

${ }^{3}$ J. D. Jones, and C. R. Fuller, "Active Control of Sound fields in Elastic Cylinders by Force Inputs,” AIAA J. 27 (7), 845-852 (1989).

${ }^{4}$ E. F. Crawley and J. de Luis, "Use of Piezoelectric Actuators as Elements of Intelligent Structures," AIAA J. 25 (10), 1373-1385 (1989).

${ }^{5}$ T. Bailey and J. E. Hubbard, "Distributed Piezoelectric-Polymer Active Vibration Control of a Cantilevered Beam," AIAA J. Guidance Control 6 (5), 605-611 (1985).

${ }^{6} \mathrm{~J}$. L. Fanson and J. C. Chen, "Structural Control by the Use of Piezoelec- tric Active Members," in Proceedings of NASA /DOD Control-Structures Interaction Conference, NASA CP-2447, Part II (1986).

${ }^{7}$ C. R. Fuller, C. H. Hansen, and S. D. Snyder, "Active Control of Structurally Radiated Noise Using Piezoceramic Actuators," Inter-Noise 89, Vol. I, 509-511 (1989).

${ }^{8}$ S. E. Burke and J. Hubbard, "Active Vibration Control of a Simply Supported Beam Using a Spatially Distributed Actuator," IEEE Control System Mag. 7 (4), 25-30 (1987).

${ }^{9}$ J. M. Plump, J. E. Hubbard, and T. Bailey, "Nonlinear Control of a Distributed System: Simulation and Experimental Results," J. Dyn. System Meas. Control 109, 133-139 (1987).

${ }^{10}$ C. K. Lee, W. W. Chiang, and T. C. O'Sullivan, "Piezoelectric Modal Sensors and Actuators Achieving Critical Damping on a Cantilever Plate," AIAA Paper No. 89-1390 (1989).

"D. W. Miller, S. A. Collins, and S. P. Peltzman, "Development of Spatially Convolving Sensors for Structural Control Applications," AIAA Paper No. 90-1127-CP, 2283-2297 (1990).

${ }^{12}$ C. K. Lee and F. C. Moon, "Modal Sensors/Actuators," ASME JAM 57, 434-441 (June, 1990).

${ }^{13}$ R. L. Clark and C. R. Fuller, "Experiments on Active Control of Structurally Radiated Sound Using Multiple Piezoceramic Actuators," J. Acoust. Soc. Am. 91, 3313-3320 (1992).

${ }^{14} \mathrm{~F}$. Fahy, Sound and Structural Vibration (Academic, Orlando, FL, 1985), pp. 60-72.

${ }^{15}$ R. A. Burdisso and C. R. Fuller, "Theory of Feed Forward Control System Eigenproperties," J. Sound Vib. 153(3), 437-452 (1992).

${ }^{16}$ Kynar Piezo Film Technical Manual (Pennwalt Corporation, King of Prussia, PA, 1987).

${ }^{17}$ C. R. Fuller, C. H. Hansen, and S. D. Snyder, "Active Control of Sound Radiation from a Vibrating Rectangular Panel by Sound Sources and Vibration Inputs: An Experimental Comparison," J. Sound Vib. 145 (2), 195-215 (1991).

${ }^{18}$ R. L. Clark and C. R. Fuller, "Optimal Placement of Piezoelectric Actuators and Polyvinylidene Fluoride (PVDF) Error Sensors in Active Structural Acoustic Control Approaches,"J. Acoust. Soc. Am. (accepted for publication ). 